\title{
Visualizing Large-Scale Uncertainty in Astrophysical Data
}

\author{
Hongwei Li, Student Member, IEEE, Chi-Wing Fu, Member, IEEE, Yinggang Li, Member, IEEE, \\ and Andrew J. Hanson, Member, IEEE
}

\begin{abstract}
Visualization of uncertainty or error in astrophysical data is seldom available in simulations of astronomical phenomena, and yet almost all rendered attributes possess some degree of uncertainty due to observational error. Uncertainties associated with spatial location typically vary significantly with scale and thus introduce further complexity in the interpretation of a given visualization. This paper introduces effective techniques for visualizing uncertainty in large-scale virtual astrophysical environments. Building upon our previous transparently scalable visualization architecture, we develop tools that enhance the perception and comprehension of uncertainty across wide scale ranges. Our methods include a unified color-coding scheme for representing log-scale distances and percentage errors, an ellipsoid model to represent positional uncertainty, an ellipsoid envelope model to expose trajectory uncertainty, and a magic-glass design supporting the selection of ranges of log-scale distance and uncertainty parameters, as well as an overview mode and a scalable WIM tool for exposing the magnitudes of spatial context and uncertainty.
\end{abstract}

Index Terms-Uncertainty visualization, large spatial scale, interstellar data, astronomy.

\section{INTRODUCTION}

Uncertainty and error are common and crucial entities in scientific studies. As pointed out by Johnson [23,24], if we look at leading peer-reviewed science and engineering journals, we can see that the majority of 2D graphs represent error or uncertainty in the experimental or simulated data. Since it is significant to show error and uncertainty in 2D graphs, it should be equally significant to show error and uncertainty in 3D visualizations as well. An assortment of uncertainty visualization methods facilitating scientific studies in different areas have been proposed in recent decades. Examples include the visualization of uncertainty in flow data $[6,30]$ and volume data $[9,27]$, positional uncertainty in molecular structure [42], uncertainty in terrain modeling $[10,50]$, and also techniques for showing uncertainty in archaeological reconstructions [46]. Being able to visualize the uncertainty in a body of data can substantially improve our understanding and interpretation of the data, and thereby facilitate better decision making.

\subsection{Motivation}

The arguments regarding the importance of error visualization are even more relevant to astrophysical data, because, for example, astrophysical measurements involve gigantic distances, so that the resultant uncertainties could typically be very large as well. For spatial quantities such as distance and velocity, characterized by standard measurements of parallax, proper motion, and radial velocity, errors can become quite significant. For instance, the Hipparcos and Tycho Catalogues [12] show that RigilKent (Alpha Centaurus, one of the closest stars to Earth in the triple star system in the constellation Centaurus) has a percentage error of $\sim 0.19 \%$, while Betelgeuse (Alpha Orionis, the brightest star in the constellation Orion) has a percentage error of $\sim 20.54 \%$ in its distance measurement. Note that RigilKent and Betelgeuse are around 4.40 and 447 light years $\left(10^{16.6} \mathrm{~m}\right.$ and $\left.10^{18.6} \mathrm{~m}\right)$ from Earth, respectively, and that the constraints of applying parallax to measure distances inevitably make the locations of the most distant stars the most uncertain.

While attention to the representation of uncertainty dominates the scientific literature on astronomical measurements, support for error

- Hongwei Li and Chi-Wing Fu are with the Hong Kong University of Science \& Technology, E-mail: $\{$ lihw,cwfu\}@cse.ust.hk

- Yinggang Li and Andrew J. Hanson are with Indiana University, Bloomington, E-mail: \{hanson,yinli\}@cs.indiana.edu

Manuscript received 31 March 2007; accepted 1 August 2007; posted online 27 October 2007. Published 14 September 2007.

For information on obtaining reprints of this article, please send e-mail to: tvcg@computer.org. representations is lacking in a large fraction of 3D astronomical visualization products. In visually spectacular animations depicting nebula flythroughs or travel from the Earth through the Milky Way Galaxy produced for planetariums and television programs, rarely does the narration or the visual representation give any hint of the huge positional uncertainty that exists in the data used to produce the rendered images. However, since, as noted in the example of Betelgeuse, the percentage error in position can be exceedingly large, and the uncertainty can significantly influence the plausibility of the visualization. The demand for pleasing visuals typically overrides the question of scientific accuracy. Our goal is to bring more attention to the importance of representing uncertainty, particularly in the presentation of $3 \mathrm{D}$ astronomical simulations.

\subsection{Focus and Organization of This Paper}

Visualizing uncertainty in astrophysical data is a unique problem. Unlike other uncertainty visualization contexts, where the distribution of data in space is generally homogeneous in nature, the distribution of astrophysical data is sparse and inhomogeneous; the farther away an object is, the harder it is to get accurate direct measurements, and the more likely it is that intervening material will confound the data. Furthermore, the scales of the data uncertainties, like astronomical distances themselves, span a huge range of scales. To address these visualization issues, we focus in this paper on improving our ability to visualize data uncertainty in large-scale environments, typified by stellar data $[4,12]$. The major objectives in our design include:

1. Providing users with a set of well-defined visual cues and user interface tools to efficiently identify and explore large-scale uncertainty in astrophysical data.

2. Properly displaying the data elements on the visual frame.

3. Enhancing the understanding of the data while minimizing the visual distraction caused by the uncertainty representations.

The paper is organized as follows: Section 2 reviews previous work on uncertainty visualizations and recent advances in astronomical visualizations. Section 3 itemizes the unique characteristics of visualizing uncertainty in astrophysical environments. Section 4 describes the unified color-coding scheme, then presents a collection of visual cues to deal with four major kinds of uncertainty in astronomical data. Section 5 then proposes our user interface tools: the magic glass design, the log-sky visualization mode, and the enhanced scalable WIM. Finally, Section 6 details our system implementation and presents a user study and a case study on the effectiveness of the proposed visualization techniques, while Section 7 gives a summary and discusses future directions. 


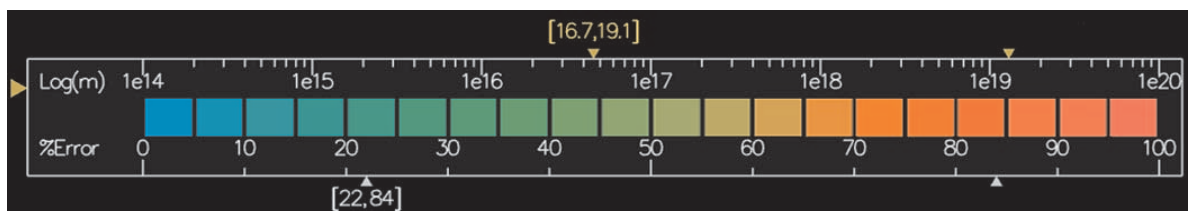

Fig. 1. A unified color-coding scheme: using colors to encode and explore large-scale spatial uncertainty and percentage errors.

\section{Related Work}

Uncertainty Visualization. Uncertainty visualization [24] was recently noted by Johnson [23] to be one of the top visualization research problems, and there is a significant body of work in the recent visualization literature. Based on the guidelines proposed by Taylor and Kuyatt [47] for evaluating data uncertainty, Lodha et al. [30,31] devised various methods for presenting uncertainty in different scenarios: among these are visual cues such as pseudo color, displacement and bump maps, displacement glyphs, and cross-hair glyphs for showing geometric uncertainty, and another set of visual techniques such as flow envelopes, twirling batons, and barbell glyphs for showing uncertainty in fluid flow. In addition, Lodha et al. [32] also experimented with the use of audio, e.g., the pitch of different musical instruments, to encode data uncertainty. Also from the same research group, Wittenbrink et al. [49] devised a new kind of vector glyph to present both the direction and magnitude uncertainty in vector fields, while Pang et al. [40] surveyed different uncertainty visualization methods, and described a systematic classification for uncertainty visualization. Djurcilov et al. [9] showed uncertainty in volume-renderings by using pseudo color, by adding noise, and by altering textures.

Subsequent work by Rhodes et al. [43] showed uncertainty in isosurfaces by mapping the uncertainty to parameters such as hue, brightness, or saturation. Grigoryan and Rheingans $[15,16]$ employed pointbased primitives to show surface uncertainty; by displacing individual surface point along its normal by an amount proportional to a random number multiplied with the local uncertainty value, a fuzzy surface was created to reveal the surface uncertainty.

Texture is another valuable element for revealing data uncertainty. Interrante [21] employed natural textures to show spatial uncertainty by distorting the regularity in natural textures; Sanderson et al. [45] explored the use of reaction diffusion to generate texture patterns with variable shapes, sizes, and orientations based on the uncertainty in the data domain; Botchen [6] subsequently developed and implemented a texture-based algorithm on the GPU to interactively show uncertainty in time-varying $2 \mathrm{D}$ flow fields.

Instead of showing the uncertainty by distorting the data or by emphasizing high uncertainty regions, Cedilnik and Rheingans [7] laid out procedurally-generated annotations on the data domain, and represented the amount of uncertainty in different areas by distorting the annotations. In the domain of information visualization, Olston and Mackinlay [38] presented the concept of bounded uncertainty, and proposed the use of ambiguation (similar to envelopes) to visually present ambiguous regions in 2D graphs and charts, while Aigner et al. [2] proposed the use of a new glyph, called PlanningLines, to show temporal uncertainty. Other recent research work in uncertainty visualization includes the work of Love et al. [33], who developed a system for visualizing a multivalued vector field so that uncertainty can be naturally incorporated in the visualization, and the work of Kniss et al. [27], who developed a comprehensive volume visualization approach that incorporates the fuzzy classification into the visualization cycle, allowing the classification decision to be deferred to the rendering stage.

Astronomical Visualization. Among relevant recent astronomical visualization work we note that of Ostriker and Norman [39], who proposed a framework for simulating cosmology and reviewed the related requirements in high performance computing environments, Nadeau et al. [14,37], who simulated a fly-through of a volumetric model of the Orion Nebula [11], Kahler et al. [26], who used a supercomputer and adaptive mesh rendering to simulate the life-span of a star, Jensen et al. [22], who devised a physically-based model to render the night sky as seen from Earth, Baranoski et al. [3], who proposed a rendering method for simulating the Aurora Borealis (the Northern Lights), Hopf et al. [20], who developed a PCA-based splatting technique for rendering point-based data in dynamic galaxy models, Magnor et al. [34, 35], who developed an inverse volume rendering method for constructing and rendering planetary nebulae and reflection nebulae, and Miller et al. [36], who derived a visualization tool to reveal structures such as filaments and voids in the Horologium-Reticulum supercluster.

In our own recent work, Hanson et al. $[17,19]$ proposed a visualization framework for illustrating the context of time in cosmological data, Li et al. [29] developed a scalable WIM (world-in-miniature) user interface for facilitating the efficient exploration of large-scale astrophysical environments, and Fu et al. [13] proposed a transparently scalable visualization architecture for modeling and rendering astronomical models across large-scale astrophysical spaces. However, it is worth noting that, except for primitive error bars constructed to show positional uncertainty, see [13], comprehensive visual representations of data uncertainty are basically absent from the bodies of astronomy visualization work mentioned thus far.

\section{Unique Characteristics: Uncertainty in Astro- PHYSICAL ENVIRONMENTS}

This section summarizes the unique characteristics of astronomical data to illustrate how the visualization of uncertainty in astrophysical environments differs from other uncertainty visualization requirements. In particular,

- First, the astrophysical space being simulated spans a very large spatial range, and yet is mostly empty; hence, users find it hard to judge distances, sizes of objects, and also the spatial context around objects in the $3 \mathrm{D}$ virtual simulations.

- Secondly, we have huge spatial ranges not only in the spatial quantities like distance and size in the astronomical data, but also in the uncertainty values. The absolute uncertainty of distances to nearby stars can range from under a light year up to several hundred light years; the vast majority of recorded stars are too distant to apply parallax methods and thus have no reliable distance assignments at all. The percentage errors can be exceedingly large compared to those typical of other scientific studies.

- Thirdly, because of the range of estimation methods and the intrinsic distances involved, some astronomical quantities can be measured with very high precision while others cannot. When locating stars, for example, we have to measure the distance to stars and the direction to stars; directions to stars can be measured with extremely high precision, while the distance measurement precision is typically much less accurate.

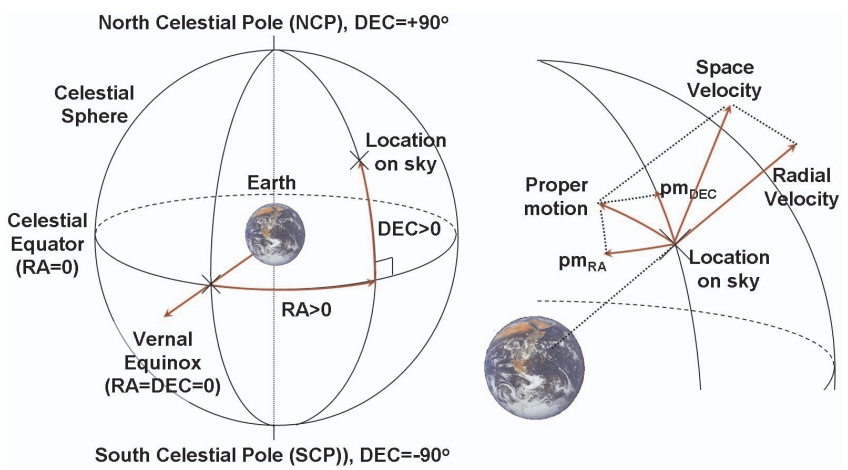

Fig. 2. The Equatorial coordinate system: $R A$ and Dec (left) and the trajectory of stars: radial velocity and proper motion (right). 

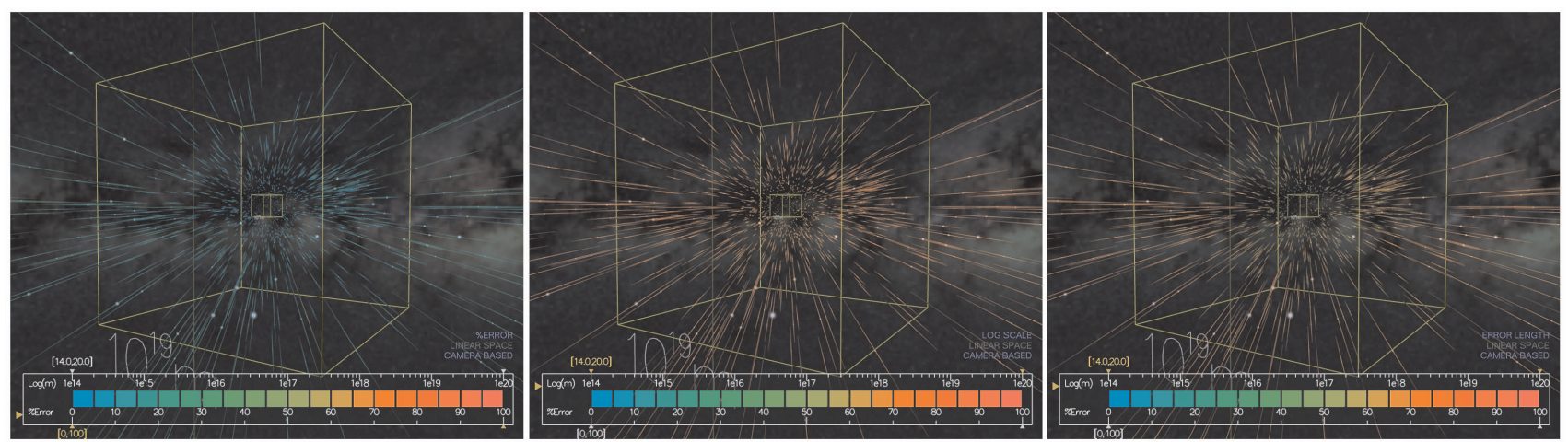

Fig. 3. Representing star positional uncertainty using error bars; from left to right, colors are assigned to annotate: 1) the percentage error of the distance uncertainty (for the most probable distance) to Earth, 2) the most probable distance, and 3) the absolute value of the distance uncertainty.

To demonstrate our proposed uncertainty visualization methods, we apply them to the following star data sets:

- The Hipparcos and Tycho Catalogues $[12,41]$. These provide star position (parallax and Equatorial coordinates $(R A, D e c)[8])$, proper motion, apparent magnitude, and color index of stars, as well as the related errors in the measurements. Note that declination $(D e c)$ is the angle measuring how far a given sky location above or below the Celestial Equator, whereas the right ascension $(R A)$ is the angle between the Vernal Equinox and the projection of the sky location on the Celestial plane, see Figure 2(left).

- The mean radial velocity catalog of galactic stars [4]. This provides radial velocities of stars (the radial component of star velocity along the line of sight) and also the associated error.

- The catalog of constellation boundary data [1].

Details on employing these data to compute a star's position, trajectory, etc., will be presented in Section 4.

\section{Visual Cues for LaRge-scale Uncertainty}

\subsection{The unified color-coding scheme}

Since the astrophysical environments we typically need to simulate are very large and very sparse, viewers have difficulty judging sizes and distances, as well as the properties of spatial uncertainty. To annotate the magnitude of spatial uncertainty in a visualization, we employ a customized unified color-coding scheme as a central design mechanism to annotate large-scale uncertainty by consistently assigning colors to the visual cues.

Color as annotation. Figure 1 shows the chosen color map; in our design, the color map can provide different kinds of annotation, specified by the user through the user interface controls. Color assignments are associated with the visual cues to represent the following:

- Large-scale distance and length: Shown on top of the color map in the figure is a base 10 logarithmic range: $\left[10^{14}, 10^{20}\right] \mathrm{m}$. This range is typically chosen to match the spatial scale in the interstellar environment, but is adjustable so that we can rescale to include galaxies or other astronomical contexts at other spatial scales. Note that this kind of annotation can be sub-categorized as: 1) the distance of a visual cue to Earth, 2) the distance of a visual cue to the camera (or viewing spacecraft), and 3) the size/length of spatial uncertainty.

- Percentage errors: Shown on the bottom of the color map in the figure is a linear range showing percentage errors.

Using Saturated Colors. The colors we employed in the color map are all fully saturated colors in the CIE L*a*b color system [48]. The advantages of this design include the following:

- When we apply these colors to shade the visual cues, the intrinsic hue of the colors will stay the same, even though their brightness may vary due to the shading. As a result, we need only very simple visual processing to estimate distances, lengths, or percentage errors; users can look solely at the hue of a given color and deduce an approximate distance or percentage error.

- Since all visual cues are colored consistently, we can visually compare uncertainty among neighboring entities by examining their color differences.

\subsection{Positional Uncertainty}

Astronomical objects in the sky are generally described by polar coordinates defining the direction to the object from Earth; for instance, we typically use Equatorial coordinates $(R A, D e c)$ for stars [8], the Galactic coordinates for objects within the Milky Way Galaxy, and the Super-Galactic coordinates to describe extra-Galactic locations.

To place objects accurately in three dimensions, we must determine the distance to the objects by various methods such as the parallax method or apparent magnitude for stars and redshift methods for galaxies. The distance $r(p, d p)$ to a star in the Hipparcos data set, as well as the standard error $d r(p, d p)$ of this distance, are determined by the parallax $p$ and its standard error $d p$. If we measure $p$ in arcseconds and choose the ideal normalization $r=1 / p$, then one arcsecond in $p$ gives a distance $r$ of 1 parsec $=3.086 \times 10^{16}$ meters. The standard but rarely-used bivariate 2 nd order expansion of the expectation of a ratio about the means given in Rice [44] reduces to the following for the case of parallax computation,

$$
\begin{aligned}
r(p, d p) & =\frac{1}{p}+\frac{d p^{2}}{p^{3}}+O\left(d p^{4}\right) \\
d r(p, d p) & =\frac{d p}{p^{2}}+O\left(d p^{3}\right),
\end{aligned}
$$

where we take $d r$ to be the square root of the variance of the distance $r$, and $d p$ to be the square root of the variance of the parallax. Since $d p$ could be relatively large with respect to $p$ in an astronomical measurement, including the $d p$ term in the expectation value of $r$ can significantly improve the computational accuracy.

Since we have uncertainty in the measurement of parallax as well as in $R A$ and $D e c$, the positional uncertainty of astronomical objects (including stars, galaxies, etc.) can always be decomposed into two components: a radial component along the line of sight (from Earth to the object) and a spherical coordinate component perpendicular to the line of sight on the Celestial sphere. To illustrate the positional uncertainty, we propose two types of visual cues:

Error bars. Due to the fact that the uncertainty in distances is several orders of magnitude larger than the uncertainty in $R A / D e c$, one way to visualize the positional uncertainty is to ignore the errors in $R A / D e c$ and plot a line segment to show the uncertainty along the line of sight from the Earth. Figure 3 represents the error bars rendered with a camera at a distance of $10^{20} \mathrm{~m}$ from Earth. Note that the positional uncertainty is, in fact, a distribution centered at the object position, and we thus draw the error bars with variable transparency; the opacity fades from one in the middle and to zero at the endpoints. Next, we use the unified color-coding scheme to shade the error bars, as noted in the figure caption. 
Ellipsoid models. The second representation we have is an ellipsoid model that emphasizes the fact that the positional uncertainty should be a volume that includes the $R A / D e c$ component. However, the uncertainty in $R A / D e c$ is exceedingly small compared to the distance uncertainty; to visualize all these uncertainty terms simultaneously, we have to artificially scale up the $R A / D e c$ uncertainty, typically by $10^{6}$. The resultant uncertainty range can be illustrated as an ellipsoid volume, see Figure 4. Note that in shading the ellipsoid model, we employ $\cos ^{-1}(\hat{V} \cdot \hat{N})$ to attenuate the surface transparency using shader programming, where $\hat{N}$ is the normal at a surface point, and $\hat{V}$ is the view direction from the surface point to the viewer. With this shading approach, we can better convey the shape of position uncertainty by emphasizing the silhouette.
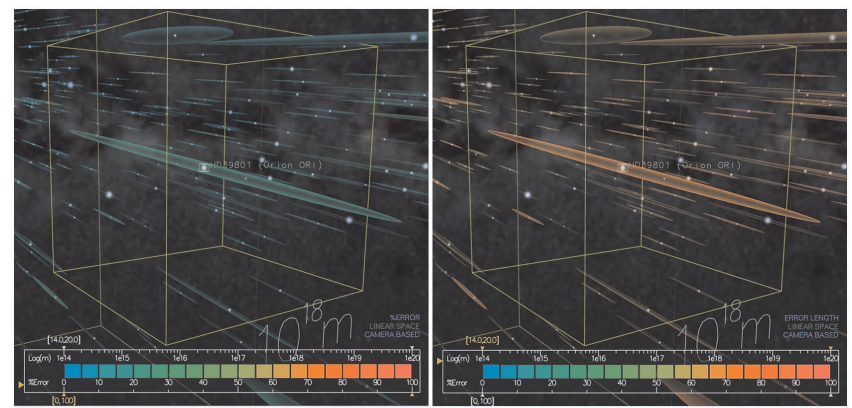

Fig. 4. Presenting positional uncertainty using ellipsoid models; coloring by percentage error (left) and by absolute error (right). Note: the chosen star is Betelgeuse: percentage error of $\sim 20.54 \%$ in its distance measurement and $\sim 447$ light years $\left(10^{18.6} \mathrm{~m}\right)$ from Earth.

\subsection{Trajectory Uncertainty}

Another common spatial quantity in astronomical observations is the trajectory or velocity (typically available for nearby stars). Like position, the velocity of astronomical objects also has two components [8]: a radial component, known as the radial velocity, along the line of sight and a component on the Celestial sphere, known as the proper motion. As depicted in Figure 2(right), we can combine proper motion and radial velocity and determine the three-dimensional velocity of any object, known as the space velocity. Note also from the figure that the proper motion has two perpendicular components: $p m_{R A}$ and $p m_{D E C}$ along the $R A$ and Dec directions, respectively. Hence, to show the positional uncertainty of the object at any moment along the trajectory path, we can employ ellipsoid models as before.
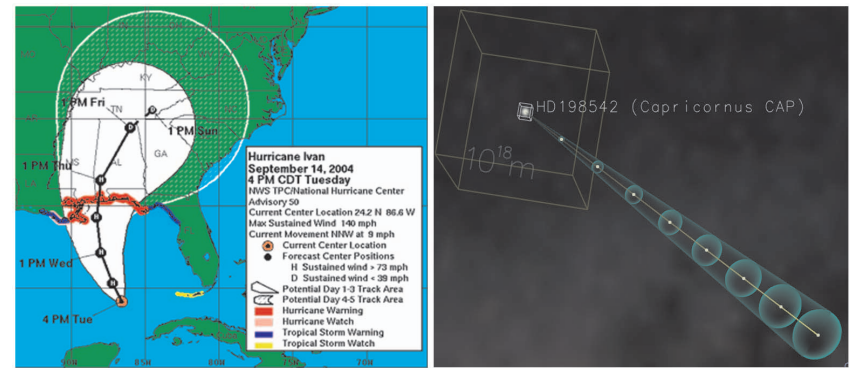

Fig. 5. Left: An example model showing trajectory uncertainty of a Hurricane. Right: The trajectory uncertainty of a star in 50,000 years; note: the color reveals the percentage error and the trajectory data of this star are 1) Radial component: $\left.(2.87 \pm 0.41) \times 10^{11} \mathrm{~m} / \mathrm{yr}, 2\right) R A$ : $(-4.34 \pm 0.57) \times 10^{-8} \mathrm{rad} / \mathrm{yr}$, and 3) Dec: $(-1.23 \pm 0.40) \times 10^{-8} \mathrm{rad} / \mathrm{yr}$.

To design the visual cues showing the trajectory uncertainty of astronomical objects, we borrow from the field of meteorology a method used to show the trajectory uncertainty of hurricane in $2 \mathrm{D}$, as shown in Figure 5. Our 3D representation for trajectory uncertainty is shown on the right hand side; we use a cone model to depict the overall trajectory uncertainty (given the time span for the trajectory) and employ an array of equally-spaced ellipsoid models to show the positional uncertainty at each time step.

\subsection{Magnitude and Color Uncertainty}

In astronomical measurements, the brightness of astronomical objects in the night sky is quantitatively defined by the magnitude scale, where this scale is applied not only to stars, but also to nebulae and galaxies. In a preliminary experiment, we attempted to visualize the magnitude uncertainty by animating the star sizes and by adding halos around stars; however, due to fact that the magnitude uncertainty was too small to be effectively perceivable using this method, we reverted to showing the uncertainty for a picked star through an auxiliary chart displayed on the user interface, as shown in Figure 6 (left) and also in Section 5. Here, we show the min-max range and also the median of the visual magnitude currently observed at the virtual viewpoint. Note that the Hipparcos and Tycho Catalogues [12] present the magnitude uncertainty as a range of visual magnitude values (as observed from Earth) together with a median term; by first converting these to absolute magnitude, we can determine the range of visual magnitude observed at any viewpoint by the following equation:

$$
m-M=5 \log _{10} r-5+A,
$$

where $M$ is the absolute magnitude, $m$ is the observed magnitude, $A$ represents the amount of light attenuation from reddening caused by the interstellar medium, and $r$ is the distance in parsecs to the astronomical object (the distance at which magnitude $m$ is measured). Note that the uncertainty in visual magnitude could change interactively as the viewpoint changes.
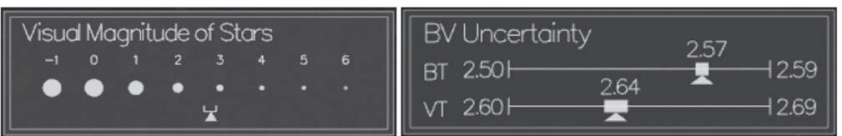

Fig. 6. Two on-screen plots of the user interface depicting the visual magnitude uncertainty (left) and B-V color uncertainty (right).

We also found that the uncertainty in color is somewhat too small to be effectively perceivable using rendering methods; therefore we also plot the uncertainty range for $B_{T}$ and $V_{T}$ as screen plots shown in Figure 6. Note that we use the Tycho photometry (available in the Hipparcos and Tycho Catalogue [12]) to represent colors: $B_{T}$ is for the blue visible range, while $V_{T}$ is for the yellow-green range; this system is closely related to Johnson UBV Photometric system [25].

\section{USER INTERFACE TOOLS}

We developed the following three major user-interface tools to support our objective of facilitating the examination and exploration of visual cues in large-scale astrophysical environments.

\subsection{The Magic Glasses}

The design of what we call "magic glasses" was motivated by the magic lens technique $[5,28]$. This interface allows users to interactively move a 2D lens over the screen to expose additional information hidden in the rendered images. In our framework, when a magic glass is put on the screen, detailed visual cues can be revealed/culled accordingly, while text labels within the glass are removed to reduce cluttering. We equip the magic glass interface with the following additional selection capability relevant to large-scale uncertainty visualization:

- Percentage error selection - Users can select a percentage error range and attach it to a magic glass; visual cues not matching this constraint do not appear in the magic glass.

- Log-scale distance selection - Users can also impose a log-scale distance constraint and select visual cues based on a log-scale distance range measured from the Earth and/or from the camera (viewpoint). Using the second type of constraint, we can equip our 2D magic glasses with proximity selection capabilities.

- Multiple magic glasses - Finally, users can deploy multiple magic glasses simultaneously. Depending on the user's choice, we can apply unions or intersections of constraints from multiple magic glasses and impose the combined constraints to select visual cues in the overlapping region. 

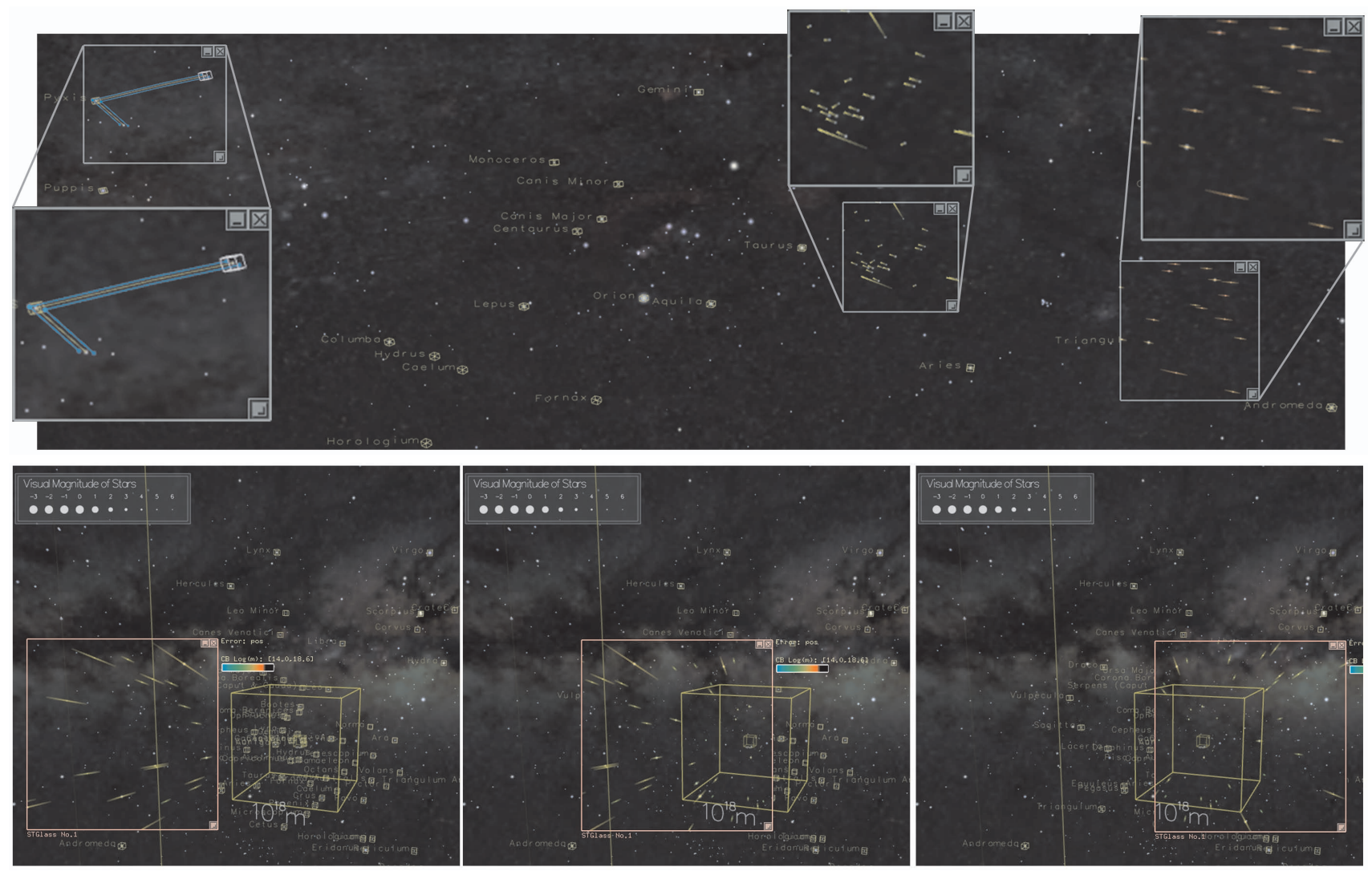

Fig. 7. Top row: A wide panoramic view centered on the Constellation Orion. Here we expose the data uncertainty using the magic glasses; Left: Positional uncertainty in constellation lines of Pyxis; Middle: Trajectory uncertainty in Taurus; Right: Positional Uncertainty. Bottom row: Moving the magic glass from left to right, exposing the properties of stars that are relatively close to us (within $10^{18.6} \mathrm{~m}$ ), while providing appropriate visual cues to characterize positional uncertainty. Color is also used to annotate the absolute size of the uncertainty.

Figure 7(top) demonstrates the use of multiple magic glasses to expose various kinds of data uncertainty in a wide panoramic view, while Figure 7(bottom) illustrates the action of moving a magic glass that selects visual cues close to the viewpoint; note the range displayed next to the label "CB $\log (\mathrm{m})$ ", where "CB" refers to camera-based logscale distance selection. Also, note that on-screen annotations such as the text labels of the constellations' major stars are removed in the magic glasses.

\subsection{The Log-Sky Visualization Mode}

Next, we define the log-sky visualization mode that provides an overview visualization by uniformly compressing the data space in a logarithmic manner. Mathematically, given a power-scaled coordinate (PSC for short), say $p=(x, y, z, s)$ (note that PSC's are defined in our previous work [13], where $p$ represents the three-dimensional position $\left.(x, y, z) \cdot 10^{s}\right)$, the log-sky visualization mode transforms $p$ using the following mapping:

$$
(x, y, z, s) \mapsto(x, y, z) \cdot f(s) \text { where } f(s)=\frac{s-a}{b-a} .
$$

Here $[a, b]$ defines the logarithmic range that the user chooses to map the data space; for example, if $a$ and $b$ are set to be 14 and 20, respectively, then we map the physical range $\left[10^{14}, 10^{20}\right]$ m logarithmically to the radial distance $[0,1]$ (measured from the sphere center) inside the unit sphere; note: the directional components $(R A / D e c)$ are kept unchanged. The advantage of this mapping is that we can efficiently utilize the space inside a unit sphere according to the typical physical range that the data covers. Note that in our original log-sky design [29] for assisting virtual exploration, $f(s)$ is basically fixed to be $s$; hence, our interstellar space, which ranges $\left[10^{14}, 10^{20}\right] \mathrm{m}$, will only cover a thin shell volume near the boundary of the unit sphere. Since this work focuses on uncertainty visualization, maximizing the space utilization can effectively improve the presentation. Figure 9 demonstrates two typical visualizations showing the positional uncertainty of stars as well as positional uncertainty in constellation lines plotted in $3 \mathrm{D}$. Note that we allow visual cues to be drawn outside the magic glass, while removing potentially cluttering text labels.

\subsection{The Scalable WIM}

In the course of data exploration in a large-scale astrophysical simulation, users typically have difficulty maintaining context awareness while focusing on the data exploration. The Scalable World-inMiniature (WIM) [29] is a powerful tool that provides users with a large-scale 3D scalable map to represent and convey the spatial context and to reduce the constraints of limited screen space. In this paper, we add visual cues and representations of uncertainty to the context of the Scalable WIM; these additional features can enhance the effectiveness of visualizing uncertainty in large-scale spaces:

- First, the Scalable WIM enables the user to examine the data uncertainty models that he/she can see on the main screen from a third-person perspective. Note that users can stay engaged in the immersive environment shown in the main window while exploring the local context in the Scalable WIM, as noted in Figure 10.

- Secondly, the Scalable WIM provides an icon-rendered view, instead of a realistically-rendered view, so that the uncertainty cues can always be more clearly seen with much less visual cluttering.

\section{IMPLEMENTATION AND RESULtS}

In our system design, the proposed uncertainty visualization techniques are implemented as a module in the transparently scalable visualization architecture [13]. To avoid concerns with scene transformation accuracy and depth buffering limits, thus supporting modeling 
and rendering of the 3D uncertainty models properly across huge spatial ranges, we represent and render all models using the PSC module architecture. In addition, to render the silhouette shading effects for the ellipsoid and cone models, we developed a shader program written in GLSL for computing the local surface transparency.

Case Study: The Distance Editing Tool. In our collaborative work with an astronomer, we have implemented a software system, known as the Distance Editing Tool [18] (DET for short), to assist her in examining metric features of materials in the local galactic neighborhood of the Sun. This system compares the distances to stars resulting from parallax measurements and photometric measurements (see the Appendix), and provides assistance in the construction and verification of geometric models for spectrally absorbing and emitting gas clouds lying between the Earth and nearby stars. To further enhance the capability of this application software, we have added modules supporting the uncertainty visualization techniques proposed in this paper and incorporated the visual cues and user-interface tools into the DET system; see Figure 11 for selected screenshots.

In addition, it is worth noting that since we deal with photometric distances in DET, we have positional (distance) uncertainty not just from parallax measurement, but also from the luminosity and spectral type classification, and B-V measurements. In the DET, we handle both of these uncertainty problems by requiring some agreement between the photometric and parallax distance. This allows us to reduce the uncertainties in the intervening ISM substantially because dust clouds can disturb the photometric distances, as could a misclassification of a star. As reported by our astronomy collaborator, requiring that photometric and parallax distances match within a designated uncertainty helped cull out misclassified spectral data.

User Study. We conducted an exploratory user study to collect feedback from users concerning their perceptions of uncertainty visualization in our system, as well as the design of the visual cues and user interface tools. Ten subjects participated in the study, three with prior experience in virtual astronomy systems; five of them are students from the graphics group and the others do not have graphics and visualization background. Six of them are males whereas the other four are females; their ages range from 23 to 28 and all of them have a normal vision and none are color blind. In doing the test, the subject worked on a single PC in an isolated room, and before that, we briefly explained the whole procedure and introduced some basic astronomy knowledge that was related; the whole test took around 30 minutes.

The first task was to examine the positional uncertainty of three stars (with distinct positional uncertainties) by checking the color on the corresponding visual cues. These data quantify the efficiency of the unified color coding scheme. In the second task, the participants were asked to locate a star among the stars with largest (based on user perception) percentage errors in positional uncertainty. Here, the ten participants are divided into two groups. One group used the range selection tool provided by the magic glass to filter out unwanted visual cues during the exploration, while the other group could use only color to do the selection. This measurement determines the relative efficiency of the range selection tool.
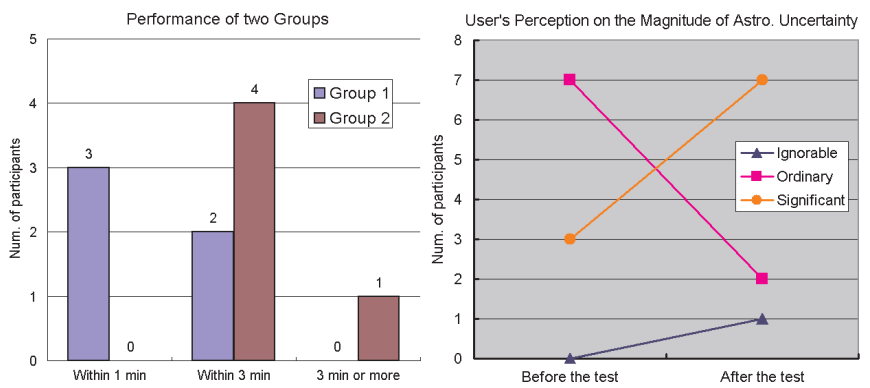

Fig. 8. Left: Performance of the two groups in locating positional uncertainty with/without range selection tool. Right: User's perception of the magnitude of astronomical uncertainty.
In the first task, the success rate of determining the percentage error (within $\pm 5 \%$ ) of the positional uncertainty (radial component) was around $96.7 \%$. This result shows that most users can efficiently employ the color coding to determine the amount of uncertainty. The users generally agreed that this was a quick and comfortable way to get an initial feeling for the amount of uncertainty; however, some users complained that they had a difficult time perceiving the details using this method and suggested using higher contrast colors for neighboring ranges in the color map. In the second task, we found that the first group (using the range selection tool) performed $64 \%$ faster than the second group (see the graph plotted on the left of Figure 8), although both groups typically succeeded in the task of locating the target star. All participants in the first group believed that the range selection tool was very important and necessary. We observed that this group of participants generally could use this tool to quickly filter out unwanted visual cues while focusing on the search for the large uncertainty. In addition, we also asked the subjects about their perceptions of the magnitude of the astronomical data, once at the beginning and once at the end. As shown on the right of Figure 8, more subjects realize the significance of considering the data uncertainty, particularly in the context of astronomical visualization, after the experiment.

\section{Conclusion}

In conclusion, we have developed an assortment of uncertainty visualization techniques designed to meet the unique challenges of largescale, sparse data typical of astronomical visualization environments. Error measures are essential components of scientifically valid astronomical visualization interfaces and are characterized by wide ranges of scale seldom encountered in other contexts. Our tools, including error encoding, custom-designed error-ellipsoids, interactive "magicglass" methods, and a scalable world-in-miniature environment are combined with power-scaled coordinates to expose astronomical error information to the user in an efficient and effective interactive manner.

Future Work We have thus far focused mainly on data such as 3D star locations from catalogs built using parallax measurements, although we have also touched on the use of photometric distance estimates. Many other large bodies of data can be incorporated into this context, including distances to nearby galaxies computed from Cepheid variable stars, distances derived from calibrated supernovae that can be used as "standard candles" in intermediate galaxies, and distances for cosmologically distant galaxies derived from redshifts. Detailed study of the error models for data such as these will result in still further unique classes of error models whose unified interactive display will support new scientific visualization capabilities.

Acknowledgments We would like to express our gratitude to our astronomy collaborator, Priscilla C. Frisch, for her tireless scientific support, suggestions, and information-checking, as well as her valuable comments on our visualization results, and also Felix Kui-Kip Lo, for his help in creating the visual cues and the video demonstration. This research was supported in part by the Research Grants Council of the Hong Kong Special Administrative Region, under RGC Earmarked Grant (Project No. HKUST612505), and also in part by NASA NAG58163, NASA NAG5-11999, and NASA NAG5-13558.

\section{REFERENCES}

[1] D. A.C. and L. S.K. Catalogue of Constellation Boundary Data, 1989. This provides data for the revised edition of "Norton's Star Atlas."

[2] W. Aigner, S. Miksch, B. Thurnher, and S. Biff. PlanningLines: novel glyphs for representing temporal uncertainties and their evaluation. In Proc. of the 9th International Conf. on Information Visualisation 2005, pages 457-463, 2005.

[3] G. V. G. Baranoski, J. G. Rokne, P. Shirley, T. Trondsen, and R. Bastos. Simulating the Aurora Borealis. In Proc. of Pacific Graphics 2000, pages 2-14, Oct. 2000.

[4] M. Barbier-Brossat and P. Figon. Catalogue général de vitesses radiales moyennes pour les étoiles galactiques. Mean radial velocities catalog of galactic stars. Astronomy and Astrophysics Supplement Series, 142:217223, Mar. 2000. Available at http://cdsweb.u-strasbg.fr/ cats/Cats.htx. 
[5] E. A. Bier, M. C. Stone, K. Pier, W. Buxton, and T. D. DeRose. Toolglass and magic lenses: The see-through interface. In Proc. of SIGGRAPH '93, pages 445-446, 1993.

[6] R. P. Botchen, D. Weiskopf, and T. Ertl. Texture-based visualization of uncertainty in flow fields. In Proc. of IEEE Visualization 2005, pages 647-654, 2005

[7] A. Cedilnik and P. Rheingans. Procedural annotation of uncertain information. In Proc. of IEEE Visualization 2000, pages 77-84, 2000.

[8] A. N. Cox. Allen's Astrophysical Quantities. Springer/Verlag, 2000.

[9] S. Djurcilov, K. Kim, P. F. J. Lermusiaux, and A. T. Pang. Volume rendering data with uncertainty information. In Data Visualization 2001, pages 243-252. Springer Verlag, 2001. Proc. of the Joint EUROGRAPHICS and IEEE TCVG Symp. on Visualization.

[10] C. R. Ehlschlaeger, A. M. Shortridge, and M. F. Goodchild. Visualizing spatial data uncertainty using animation. Computers \& Geosciences, 23(4):387-395, May-Jun. 1997.

[11] C. Emmart, D. R. Nadeau, J. Genetti, and E. Wesselak. Volume Visualization of the Orion Nebula, 2000. SIGGRAPH 2000 Electronic Theatre, Issue 134: http: / /vis.sdsc.edu/research/orion.html.

[12] European Space Agency. The Hipparcos and Tycho Catalogues, ESA SP-1200, 1997. Available at http://www.rssd.esa.int/ Hipparcos/catalog.html.

[13] C.-W. Fu and A. J. Hanson. A transparently scalable visualization architecture for exploring the Universe. IEEE Tran. on Visualization and Computer Graphics, 13(1):108-121, 2007.

[14] J. D. Genetti. Volume-rendered galactic animations. Communications of the ACM, 45(11):62-66, 2002.

[15] G. Grigoryan and P. Rheingans. Probabilistic surfaces: Point based primitives to show surface uncertainty. In Proc. of IEEE Visualization 2002, pages 147-154, 2002.

[16] G. Grigoryan and P. Rheingans. Point-based probabilistic surfaces to show surface uncertainty. IEEE Tran. on Visualization and Computer Graphics, 10(5):564-573, Sep 2004

[17] A. J. Hanson and C.-W. Fu. Cosmic Clock, 2000. Siggraph Video Review, vol. 134 , scene 5 .

[18] A. J. Hanson, C.-W. Fu, and P. C. Frisch. Constraint-based astronometric modeling tools. In G. Brunnett, B. Hamann, and H. Mueller, editors, Geometric Modeling for Scientific Visualization, pages 437-452. SpringerVerlag, 2003.

[19] A. J. Hanson, C.-W. Fu, and E. A. Wernert. Visualizing cosmological time. In F. H. Post, G. M. Nielson, and G.-P. Bonneau, editors, Data Visualization: The State of the Art, pages 423-438. Kluwer academic publishers, May 21-26, 2000, Dagstuhl, DE, 2003.

[20] M. Hopf, M. Luttenberger, and T. Ertl. Hierarchical splatting of scattered 4D data. IEEE Computer Graphics and App., 24(4):64-72, 2004.

[21] V. Interrante. Harnessing natural textures for multivariate visualization. IEEE Computer Graphics and App., 20(6):6-11, Nov.-Dec. 2000.

[22] H. W. Jensen, F. Durand, J. Dorsey, M. M. Stark, P. Shirley, and S. Premoze. A physically-based night sky model. In Proc. of ACM SIGGRAPH 2001, pages 399-408, Aug. 2001.

[23] C. R. Johnson. Top scientific visualization research problems. IEEE Computer Graphics and App., 24(4):13-17, 2004.

[24] C. R. Johnson and A. R. Sanderson. A next step: Visualizing errors and uncertainty. IEEE Computer Graphics and App., 23(5):6-10, 2003.

[25] H. L. Johnson and W. W. Morgan. Fundamental stellar photometry for standards of spectral type on the revised system of the Yerkes spectral atlas. The Astrophysical Journal, 117:313-352, 1953.

[26] R. Kahler, D. Cox, R. Patterson, S. Levy, H.-C. Hege, and T. Abel. Rendering the first star in the Universe - a case study. In IEEE Visualization 2002, pages 537-540, 2002.

[27] J. M. Kniss, R. V. Uitert, A. Stephens, G.-S. Li, T. Tasdizen, and C. Hansen. Statistically quantitative volume visualization. In Proc. of IEEE Visualization 2005, pages 287-294, 2005.

[28] E. LaMar, B. Hamann, and K. I. Joy. A magnification lens for interactive volume visualization. In Proc. of the Ninth Pacific Conf. on Computer Graphics, pages 223-232, 2001.

[29] Y. Li, C.-W. Fu, and A. J. Hanson. Scalable WIM: Effective exploration in large-scale astrophysical environments. IEEE Tran. on Visualization and Computer Graphics, 12(5):1005-1011, 2006.

[30] S. K. Lodha, A. T. Pang, R. E. Sheehan, and C. M. Wittenbrink. UFLOW: visualizing uncertainty in fluid flow. In Proc. of IEEE Visualization 1996, pages 249-254, 1996.

[31] S. K. Lodha, B. Sheehan, A. T. Pang, and C. M. Wittenbrink. Visualizing geometric uncertainty of surface interpolants. In Proc. of the conf. on Graphics interface '96, pages 238-245, 1996.

[32] S. K. Lodha, C. M. Wilson, and R. E. Sheehan. LISTEN: sounding uncertainty visualization. In Proc. of IEEE Visualization 1996, pages 189-195, 1996.

[33] A. L. Love, A. T. Pang, and D. Kao. Visualizing spatial multivalue data. IEEE Computer Graphics and App., 25(3):69-79, May-Jun. 2005.

[34] M. Magnor, K. Hildebrand, A. Lintu, and A. J. Hanson. Reflection nebula visualization. In IEEE Visualization 2005, pages 255-262, Oct. 2005.

[35] M. Magnor, G. Kindlmann, and C. Hansen. Constrained inverse volume rendering for planetary nebulae. In IEEE Visualization 2004, pages 83 90 , Oct. 2004.

[36] J. Miller, C. W. Quammen, and M. C. Fleenor. Interactive visualization of intercluster galaxy structures in the horologium-reticulum supercluster. IEEE Tran. on Visualization and Computer Graphics, 12(5):1149-1156, 2006.

[37] D. R. Nadeau, J. D. Genetti, S. Napear, B. Pailthorpe, C. Emmart, E. Wesselak, and D. Davidson. Visualizing Stars and Emission Nebulae. Computer Graphics Forum, 20(1):27-33, Mar. 2001. Also in Eurographics 2000 (short presentations).

[38] C. Olston and J. D. Mackinlay. Visualizing data with bounded uncertainty. In Proc. of the IEEE Symposium on Information Visualization, pages 37-40, Oct. 2002.

[39] J. P. Ostriker and M. L. Norman. Cosmology of the early Universe viewed through the new infrastructure. Communications of the ACM, 40(11):84 94, Nov. 1997.

[40] A. T. Pang, C. M. Wittenbrink, and S. K. Lodha. Approaches to uncertainty visualization. The Visual Computer, 13(8):370-390, Nov. 1997.

[41] M. Perryman et al., editor. The Hipparcos and Tycho Catalogues. ESA Publications Division, The Netherlands, 1997.

[42] P. Rheingans and S. Joshi. Visualization of molecules with positional uncertainty. In Proc. of Data Visualization 1999, pages 299-306, 1999.

[43] P. J. Rhodes, R. S. Laramee, R. D. Bergeron, and T. M. Sparr. Uncertainty visualization methods in isosurface rendering. In EUROGRAPHICS 2003 Short Papers, pages 83-88, Sep. 2003.

[44] J. A. Rice. Mathematical Statistics and Data Analysis. Duxbury Press, 1994. 2nd edition.

[45] A. R. Sanderson, C. R. Johnson, and R. M. Kirby. Display of vector fields using a reaction-diffusion model. In Proc. of IEEE Visualization 2004, pages 115-122, 2004.

[46] M. Sifniotis, B. Jackson, M. White, K. Mania, and P. Watten. Visualising uncertainty in archaeological reconstructions: a possibilistic approach, 2006. Technical Sketch at ACM SIGGRAPH 2006.

[47] B. N. Taylor and C. E. Kuyatt. Guidelines for evaluating and expressing the uncertainty of NIST measurement results, 1994. Technical report, National Institute of Standards and Technology Technical Note 1297, Gaithersburg, MD, 1994 Edition.

[48] C. Ware. Information Visualization: Perception for Design. Morgan Kaufmann, 2004. 2nd edition.

[49] C. M. Wittenbrink, A. T. Pang, and S. K. Lodha. Glyphs for visualizing uncertainty in vector fields. IEEE Tran. on Visualization and Computer Graphics, 2(3):266-279, Sep. 1996.

[50] J. D. Woods and P. F. Fisher. Assessing interpolation accuracy in elevation models. IEEE Computer Graphics and App., 13(3):48-56, 1993.

\section{Appendix: Computing Photometric Distances}

While parallax measurement is the standard method for determining the distance to nearby stars, alternatives are available. One such method employs stellar photometry data, and is based on the relationship between stellar distance and the difference between the measured visual magnitude and the estimated absolute magnitude, as given in Equation 3. In this approach, we first determine the absolute magnitude of a star by using a well-established table in [8], "Calibration of MK spectral types." This table maps the observed spectral type, spectral class, and spectral number of a star to the expected absolute magnitude value. Using an astronomer's rule of thumb, we set the reddening term $A$ to be $3.1 \times E(B-V)$, where 3.1 is a general constant representing a wide range of the interstellar medium. We can then invert Equation 3 to compute the distance to a star. Note: $E(B-V)$ is the color excess, i.e., the difference between the measured $B-V$ color index and the intrinsic $B-V$ color index given in the cited table for the absolute magnitude in [8]. 

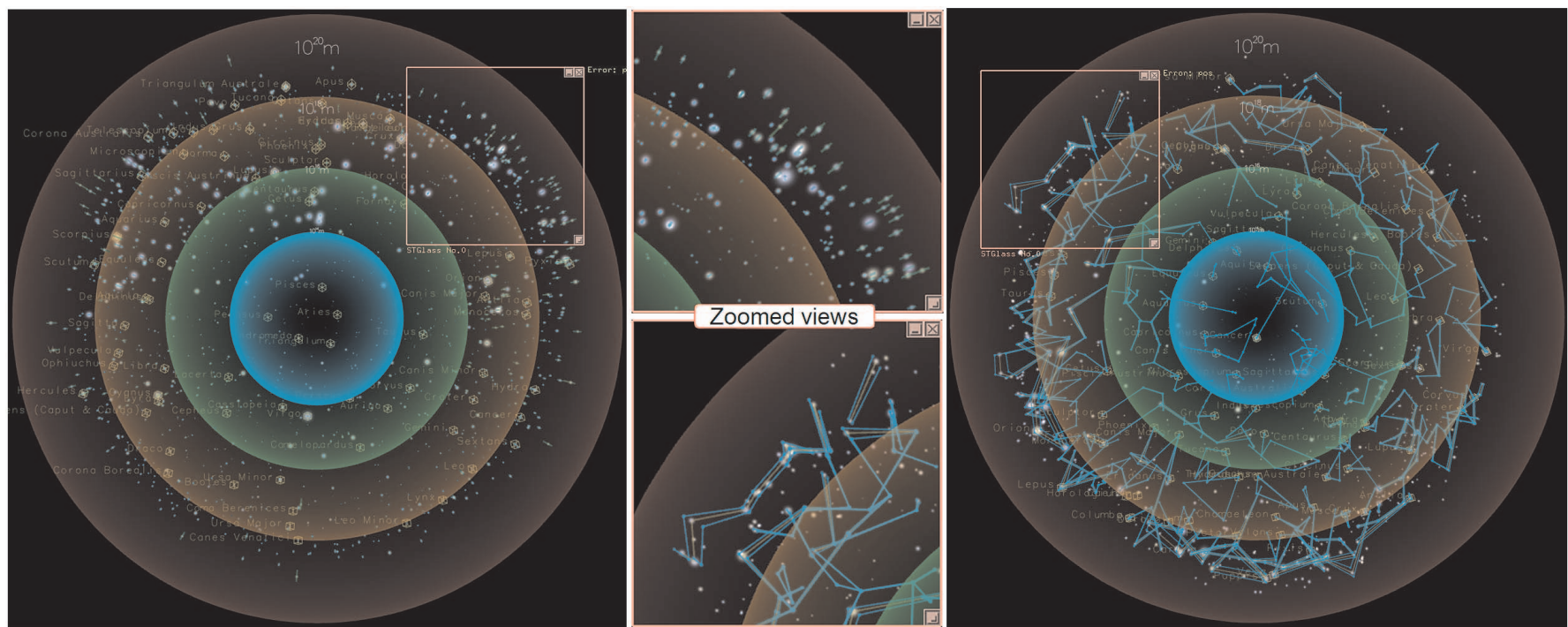

Fig. 9. The log-sky visualization mode; Left: positional uncertainty (error bars) of stars; Right: positional uncertainty of constellation lines plotted in 3D; Middle: two zoomed views.

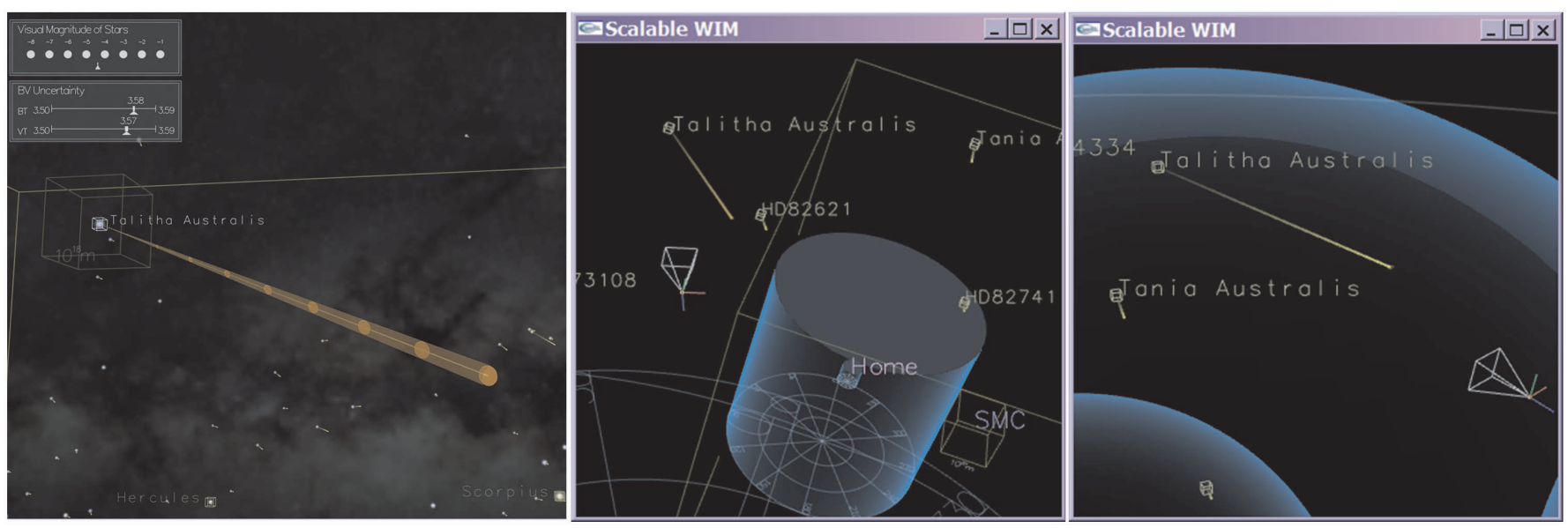

Fig. 10. Using the Scalable WIM to explore and examine data uncertainty from a third-person viewpoint; Left: main window showing a trajectory uncertainty; Middle and Right: the Scalable WIM, as a popup window, exposes the spatial context around the uncertainty model.

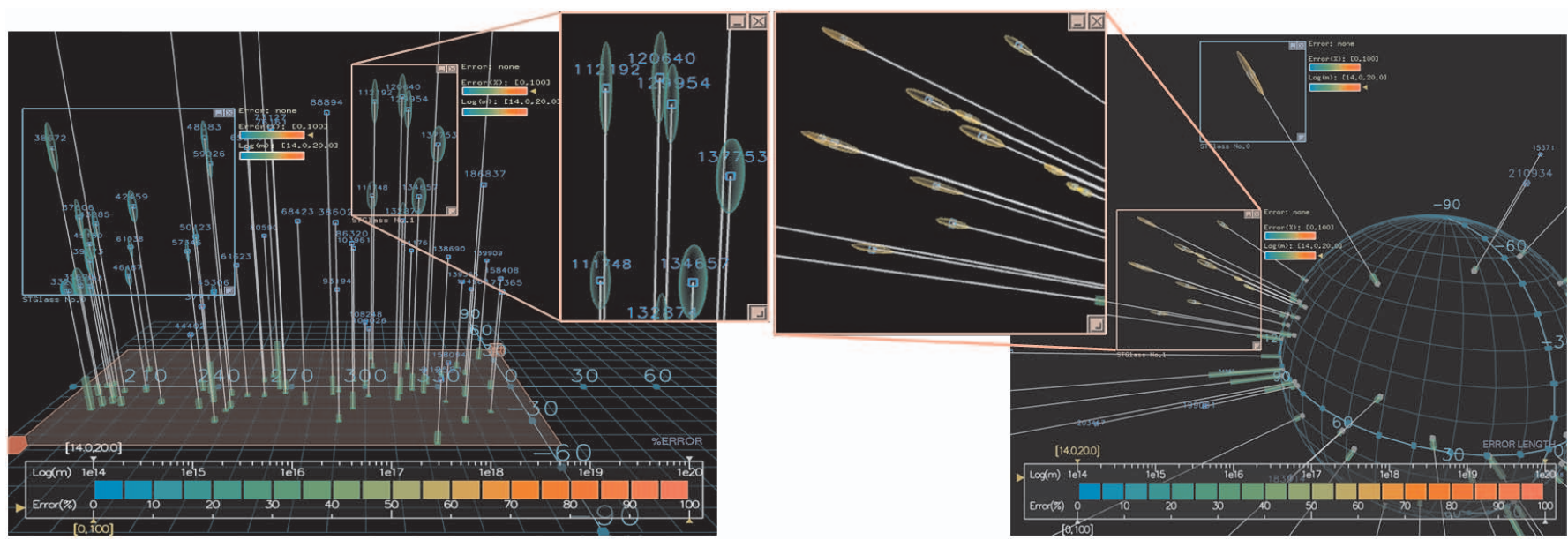

Fig. 11. Uncertainty visualization in the DET: Ellipsoid models show positional uncertainty and magic glasses expose uncertainty. The DET supports two modes: 1) Planar mode (left) (the ground plane is a rectangular space corresponding to RA/Dec coordinates) and 2) Sphere mode (right) (projected onto the Celestial sphere). Note: we can hide the text labels inside the magic glasses to avoid information cluttering (right). 